\title{
Urbanité ludique et cultures sportives : analyse de quatre pratiques physiques de l'espace parisien
}

\section{Florian Lebreton \& Stéphane Héas}

To cite this article: Florian Lebreton \& Stéphane Héas (2010) Urbanité ludique et cultures sportives : analyse de quatre pratiques physiques de l'espace parisien, Loisir et Société / Society and Leisure, 33:2, 195-219, DOI: 10.1080/07053436.2010.10707809

To link to this article: https://doi.org/10.1080/07053436.2010.10707809

曲 Published online: 02 Jul 2013.

Submit your article to this journal

Џ Article views: 40

Citing articles: 1 View citing articles $\widetilde{ }$ 


\title{
URBANITÉ LUDIQUE ET CULTURES SPORTIVES: ANALYSE DE QUATRE PRATIQUES PHYSIQUES DE L'ESPACE PARISIEN
}

\author{
Florian LEBRETON \\ Stéphane HÉAS
}

Université européenne de Bretagne - Rennes II

\section{Introduction}

Les pratiques physiques analysées ici (spéléologie urbaine, base-jump ${ }^{1}$, parkour $^{2}$ et street golf) contribuent à élargir, entre autres, un peu plus l'éventail des cultures sportives et urbaines ${ }^{3}$. Ces usages ludiques participent-ils d'une subculture sportive en tant que catégorie marginale? Ce concept de subculture est souvent employé pour décrire une activité émergente sans chercher à en comprendre les logiques et modalités plurielles. Des ethnographies sportives nord-américaines ont souligné ce point (Donnelly, 1985, 1993; Atkinson et Young, 2008). Nous nous sommes intéressés à la question des pratiques ludo-sportives urbaines sous l'angle des cultures de rue et de leurs impacts sur la reformulation des cultures urbaines et sportives ${ }^{4}$. Au-delà de leur caractère éminemment masculin, des différences majeures apparaissent à l'intérieur des pratiques urbaines. Si elles sont situées en marge des institutions sportives traditionnelles - elles sont pour la plupart auto-organisées -elles n'en sont pas pour autant totalement exclues. En jouant sur le caractère visible versus caché des espaces pratiqués, les jeux des acteurs montre une bipolarisation entre, d'un côté, le désir d'être reconnu publiquement pour ce qu'ils font et, de l'autre côté, le refus de médiatiser l'activité. Ainsi, il s'agit pour nous d'approfondir la question de la dimension utopique et distinctive des activités émergentes 
sur l'espace public dans la mesure où elles investissent l'espace urbain, sortent l'activité physique des installations ad hoc et agissent en dehors d'un espace professionnalisé. Quel est notre objectif ici? Les pratiques urbaines, comme toutes les pratiques culturelles sont en perpétuelle effervescence. Cherchons à interroger l'incorporation d'une (sous)culture à travers des territorialités urbaines. Selon nous, ces pratiques sont non seulement inscrites dans la ville, mais sont d'abord de la ville. En somme, le contexte urbain, la ville, ses architectures ou encore ses modes de vie les produisent largement. Pourquoi, comment et sous quelles conditions la ville les produit-elle? Cette recherche illustre d'une manière originale l'urbanité parisienne où la rue devient un lieu de socialisation par l'activité physique alternative.

Les quatre pratiques ethnographiées répondent à une logique commune: «faire vivre la ville» (dixit un enquêté), pour la rendre agréable à vivre et surtout, apprendre à l'aimer. Le projet de ces pratiquants est d'animer une urbanité ludique, c'est-à-dire de réaliser une quête hédoniste des espaces urbains qui contribue alors à requalifier sociologiquement la ville. Cette manière d'aborder la ville ne redonne-t-elle pas à l'espace public un esprit fédérateur? Alors, les activités de rues analysées ici apparaissent largement comme des lieux de socialisation trop souvent sous-estimés par les acteurs institutionnels. Ces ethnographies apportent un regard nouveau sur la question des cultures urbaines et sportives car elles illustrent l'émergence des activités dites informelles et auto-organisées, en marge des réseaux traditionnels de sociabilités (associations sportives, clubs sportifs, etc.) (Héas et Saïdi, 2000). Les «traceurs $»^{5}$, les street golfeurs, les spéléologues et les base-jumpers urbains entretiennent une relation ludique avec l'environnement urbain. Les escaliers, les rues, les souterrains, les immeubles, les places, les jardins, les tours et les parcs publics deviennent des terrains de jeu où chacun entre en scène. Soumis à l'appréciation des pairs (le plus souvent des hommes), chaque pratiquant joue individuellement son rôle pour s'approprier collectivement le lieu fréquenté, usé, marqué et pratiqué par le groupe masculin. L'urbanité ludique s'interprète ainsi, à travers ce jeu d'appropriation spatiale et sensorielle. En revanche, il est possible de différencier ces cultures urbaines selon qu'elles s'inscrivent sur des espaces visibles ou bien invisibles de Paris. Cette première dissemblance culturelle distingue ces pratiques urbaines puisque la volonté de se faire voir prévaut pour certains - le parkour et le street golf, alors que pour d'autres, la culture du secret et de la «clandestinité urbaine» (Aprile et Retaillaud-Bajac, 2008) importe le plus. 


\section{Des pratiques qui s'inscrivent sur l'espace urbain}

La particularité de ces terrains de recherche tient dans l'absence de travaux antérieurs, excepté pour le parkour et le base-jump où une très nette augmentation des publications sur le sujet émerge d'Angleterre, des États-Unis et du Canada plus récemment. La sociologie française ne s'est pas suffisamment intéressée à cette «nouvelle » pratique si ce n'est l'étude anthropologique de Calogirou (1999). À notre connaissance, l'auteure est la première à avoir intégrer le parkour dans ses recherches menées sur les sports urbains. Elle présente l'activité inconnue jusqu'alors, où «le sportif utilise les éléments urbains pour réaliser un parcours d'obstacles au gré de son cheminement et des objets urbains et constructions qu'il rencontre » (op. cit.). Une série d'études pluridisciplinaires sur le parkour a émergé lors des trois dernières années. Une grande partie de ces travaux explore cette «nouvelle » forme de mouvement comme une façon d'interagir avec l'environnement urbain et le milieu de vie (Geyh, 2006). Ces interactions ludiques ont pour fonction de défier plutôt que de contester les usages et significations des espaces urbains, la vie urbaine et les formes urbaines de gouvernement des corps (Atkinson, 2009; Bavinton, 2007; Saville, 2008; Thompson, 2008; Mould, 2009). Pour d'autres, le parkour transgresse l'espace urbain capitaliste, perturbe et déstabilise les significations capitalistes propres aux villes postindustrielles (Daskalaki, Stara et Miguel, 2008). Le parkour apparaît alors comme une forme d'« activisme urbain» qui se propose de défier les activités sociales contemporaines (Daskalaki, Stara et Miguel, 2008, p. 61). Dans le même registre, Atkinson (2009) souligne que les pratiquants ethnographiés à Toronto défient un environnement urbain aseptisé et ainsi, selon l'auteur le parkour devient une forme contemporaine de «mouvement anarcho-environnemental». Que retenir ici? Le modèle du plaisir et de la participation active des pratiquants dans leurs propres communautés de pratique illustre l'idée selon laquelle le parkour, la spéléologie urbaine, le base-jump urbain et le street golf fonctionnent comme un «échappatoire aux pratiques du pouvoir qui régissent nos mouvements et gouvernent nos comportements » (Ortuzar, 2009, p. 55).

Sur un autre registre, la pratique de la spéléologie urbaine est quasi absente de la littérature sociologique française mais également anglosaxonne. Néanmoins, nous retenons qu'une première enquête ethnologique avait eu lieu au début des années 1980 par B. Glowczewski ${ }^{6}$ (1983).

Le base-jump, essentiellement montagnard, fait l'objet de quelques analyses sociologiques et psychologiques. Sur le même mode opératoire que le nôtre (observations et entretiens), Martha et Griffet (2006) ont analysé la pratique dans le sud-est de la France. Qualifié de «jeu le plus sérieux au 
monde», l'activité combine le sérieux et le jeu, ce qui procure une "expérience aventureuse». Quant aux travaux anglo-saxons, on peut retenir ceux de B. Wheaton (2004) où le base-jump est un «style de vie» mettant en avant les notions de sensations et de la mise en mouvement du corps dans l'espace. Quant au saut urbain, nous n'avons pas recensé de travaux sur ce point. Et pourtant, une question fondamentale surgit de cette pratique: comment interpréter la relation entre le corps en mouvement dans la chute et l'inscription de celui-ci au cœur des cités urbaines?

À notre connaissance, le street golf ne fait pas encore l'objet d'analyse sociologique. Il n'existe donc aucune référence précise sur le sujet si ce n'est le grand nombre d'articles journalistiques. Il est d'ailleurs intéressant de noter que le street golf n'a pas encore traversé l'Atlantique puisque cette pratique est principalement centrée en Europe (Allemagne, France).

Bien qu'elles soient pratiquées en France depuis les années 1990 et 2000 , ces deux dernières pratiques ne sont pas encore populaires auprès du grand public et, à notre sens, ne sont pas suffisamment traitées au regard des questions sociétales ainsi soulevées. En s'inscrivant au cœur des grandes villes, elles marquent les cultures urbaines de leurs empreintes respectives.

Inspiré par la «méthode naturelle» de G. Hébert (1942) - méthode d'entraînement du corps, le parkour est aujourd'hui une activité physique et sportive reproduisant certains exercices corporels sur les mobiliers urbains. L'objectif est la déambulation «libre» à travers la ville pour y exécuter des mouvements gymniques et athlétiques le tout en harmonie avec les architectures et l'environnement urbain. Les traceurs, nom que l'on attribue aux pratiquants, ont alors inventé un ensemble de gestes techniques adaptés à l'environnement urbain. Le tableau suivant recense quelques figures pour le moins évocatrices de cette activité urbaine.

Le street golf illustre, quant à lui, parfaitement la transformation culturelle des APS contemporaines car il est une adaptation du golf traditionnel à l'environnement urbain. Axée sur la déambulation dans la ville, le jeu consiste à jouer sur le bitume avec une balle de type semi-rigide et des fers spécifiques. Le mobilier urbain devient ainsi une cible sur laquelle les pratiquants se concentrent: poubelles, jardins publics, bancs publics, panneaux de signalisation, etc. L'espace de jeu est lui aussi inspiré par l'environnement urbain, il n'est donc jamais figé, il se réinvente perpétuellement au hasard des déambulations urbaines. En d'autres termes le street golf, prenant parfois le nom d'urban golf ou encore de cross golf, est une mutation du golf traditionnel qui s'est orientée vers un des plus grands terrains de sport: la rue.

D’une manière générale, il est possible de repérer quelques traits structurant ces pratiques urbaines. Une socialisation masculine domine les activités physiques et sportives dites auto-organisées et conduit alors 


\section{TABLEAU 1}

\section{De quelques figures spécifiques du parkour}

\begin{tabular}{ll} 
Nom de la figure & Description \\
«saut de chat» & $\begin{array}{l}\text { En prenant appui avec les mains, sauter un obstacle } \\
\text { en passant les jambes entre les mains. }\end{array}$ \\
\hline «saut de fond» & $\begin{array}{l}\text { Se réalise par une poussée vers l'avant dans le vide } \\
\text { nécessitant une réception sur les pieds. Le franchissement } \\
\text { s'achève par une roulade. }\end{array}$
\end{tabular}

«saut de bras»

Basé sur un saut, il s'agit ici de réceptionner avec les mains, les bras ou le coude pour saisir le bord d'un mur.

«passe-muraille» Technique de franchissement d'un mur d'une hauteur assez importante: consiste à prendre appui sur le mur avec un pied pour atteindre une plus grande hauteur afin de saisir une prise pour passer l'obstaclea.

$\begin{array}{ll}\text { «saut de précision» } & \begin{array}{l}\text { C'est la maitrise de l'atterrissage, la cible, un point fixe } \\ \text { de petite taille, qui nécessite une évaluation fine du saut } \\ \text { pour atterrir précisément }\end{array}\end{array}$

$\begin{array}{ll}\text { «tic-tac» } & \text { Technique de franchissement d'un petit obstacle. } \\ \text { En courant, on place un pied sur le mur et on l'emploie } \\ \text { comme équilibre pour se propulser et franchir } \\ \text { l'obstacle suivant. }\end{array}$

«lâcher»

Se lâcher d'une hauteur en chute libre, pour se réceptionner sur les jambes ou rattraper une autre prise.

«balancer» Suspendu à l'aide des mains sur une barre, il s'agit de lancer le corps d'avant en arrière afin de se lâcher pour rattraper une autre prise ou exécuter une réception.

«wallflip» Prendre appui avec un appel du pied sur un mur, puis faire un salto avant ou arrière à $360^{\circ}$.

«planche»

Technique pour monter sur un plan où vous tenez les bras au-dessus de l'obstacle. Placer les mains à hauteur de l'obstacle, prendre appuie, puis élever le reste du corps à la force des bras pour monter vers le hautc .
a) Cette technique de franchissement n'est pas sans rappeler sa consœur militaire dans le dénommé "parcours du combattant»
b) Proche du saut de précision au parachutisme par exemple.
c) Technique utilisée fréquemment dans les tests physiques des pompiers professionnels. 
à une exclusion, symbolique, de la femme car elles sont faiblement représentées au sein de ces communautés pratiquantes, nous avons pu nous en rendre compte durant nos ethnographies. Un second trait semble structurer ces communautés: l'âge mais surtout l'environnement urbain. Les pratiquants appartiennent généralement aux catégories adolescentes et jeunes adultes, ce qui corrobore les travaux sur les «styles de vie sportifs» (Wheaton, 2004), et sont très largement imprégnés des cultures urbaines (musique, sports), des nouvelles technologies (médias de diffusion, internet), bref, une appartenance au milieu urbain très prononcé. En revanche, le base-jump urbain se démarque des trois autres pratiques car il s'agit d'une pratique, initialement, «montagnarde» mais transposée au milieu urbain et à l'environnement quotidien de quelques sauteurs.

\section{La méthode socio-anthropologique}

Comprendre ces comportements ludiques revient à interroger le rapport au jeu et à l'espace par le corps, aussi bien au regard des pratiques que des représentations et valeurs qu'il recouvre. Il s'agit d'identifier les fondements d'un possible «exister ensemble» à la fois sportif, ludique et festif. À l'instar de Bromberger pour qui une démarche de ce type doit s'appuyer pour l'essentiel sur une «enquête qualitative, l'écoute situationnelle [...] l'observation plus ou moins participante, l'attention aux détails, aux dimensions sensibles de l'expérience» (2004, p. 119), nous avons retenu deux principes essentiels. Le premier questionne l'intérêt de s'engager plus ou moins pleinement sur le terrain. Il est mobilisé ici par le recours aux entretiens exploratoires dans un premier temps (via le Net et l'intrusion dans les «réseaux virtuels » présents sur la toile), puis aux entretiens plus approfondis $(\mathrm{N}=34)$ par la suite, le recours à l'observation minutieuse (participante et/ou distanciée), la rédaction d'un carnet ethnographique, puis l'étude de documents divers appartenant aux pratiquants et aux différents groupes et, enfin, un suivi des articles dans la presse locale, régionale et nationale sur le sujet. Cette première phase est bien entendue complétée par des moments de distanciation propices à une écriture réflexive.

La difficulté de cette démarche réside dans la position du chercheur, qui oscille entre empathie et immersion mesurée. Les observations participantes font alors figure de jeux interactifs entre «eux» et «nous», car elles sont constituées de conversations, de gestes, de jeux de regards, etc. Ce jeu méthodologique permet de négocier en permanence la place de l'enquêteur principal au sein des groupes enquêtés, soit un ensemble de tactiques mises en place par le chercheur négociant continuellement sa place dans la société ethnographiée (Piette, 1996). 


\section{Un sociologue en ville!}

Le travail de terrain, à la fois participant ${ }^{7}$ et distancé, permet d'appréhender «la société telle qu'elle est perçue du dedans par les acteurs sociaux» (Laplantine, 1996, p. 20). Soit, une tentative pour «s'établir au plus près des altérités en présence [...] se situer dans un équilibre instable entre empathie et différence afin d'établir un rapport où l'Autre a des possibilités réciproques de dialoguer, de connaissances» (Bouvier, 2000, p. 70). Cette démarche réciproque incite volontiers à circuler entre le point de vue emic (acteur/usager) et le point de vue etic (observateur) (Bromberger, 2004). Cette posture réflexive de l'observateur relève d'une démarche inductive et délibérative (Mukamurera, 2006), sous peine de glisser vers une «auto-ethnographie» dangereuse à bien des égards. La socio-anthropologie distingue notamment une observation impliquée et une autre distanciée (Bouvier, 1995). Or, ces deux pôles (engagement/distanciation) s'articulent et ne sont pas isolés (Elias, 1993; Chevalier et Privat, 2004; de Sardan, 2008). À travers ce double processus, le projet socio-anthropologique prend toute sa valeur.

La place du chercheur en ville - et plus particulièrement de l'ethnologue et du sociologue - n'est pas nouvelle (Ostrowetsky, 1996). En revanche, la compréhension de communautés d'individus - plus ou moins secrètes - qui pratiquent les espaces souterrains ${ }^{8}$ et urbains $^{9}$ (parcs, jardins, immeubles, rues, escaliers, poubelles, etc.) sur le mode de la transgression l'est beaucoup moins. Dans le cadre de notre «première» recherche, de nombreuses questions en matière de déontologie se posaient: comment procéder pour enregistrer un maximum d'informations sans glisser vers une empathie exagérée ni trahir la confiance que ces acteurs nous accordaient? Ces terrains à prospecter interrogent chacun des habitants de la ville dans ses relations quotidiennes avec elle. Ces terrains mêlent deux échelles croisées et imbriquées. Pour la première échelle macrosociologique, la ville dépend directement des grandes structures économiques, politiques, culturelles et sociales qui participent à une «intensification de la vie nerveuse» (Simmel, 2004). Le trait dominant de la vie urbaine et moderne questionne les sociabilités, les processus d'impersonnalités, d'individualisations et même d'intellectualités (Joseph, 1998). La ville demeure le lieu des masques, des rôles sociaux, voire des rôles de représentations théâtrales sur le modèle théorique et métaphorique de Goffman. À cette première échelle de la réalité sociale et urbaine s'oppose une autre soucieuse du vécu humain, davantage microsociologique. Des microgroupes d'individus parcourent la ville à la recherche d'espaces qui seraient potentiellement transformables et malléables en espaces de corporéités ludiques et sportives. Dans cette optique, la ville devient un espace à découvrir, à explorer et à pratiquer (Hannertz, 1983). 
Comment ces deux échelles s'interpénètrent? Comment accéder aux significations développées par les enquêtés? Sommes-nous en présence d'un univers polyphonique et polyrythmique en ville (Sansot, 1986, 2004)? Les discours et les représentations sur la ville contemporaine montrent en effet sa possible requalification sociologique et anthropologique dans la mesure où elle n'est plus ce qu'elle était! La ville est perpétuellement à la merci de ce qui bouge, de ce qui s'invente et de ce qui se multiplie au quotidien par et à travers la diversité culturelle des pratiques sociales.

\section{La question du choix et du lieu des activités}

Nous avons ethnographié les quatre pratiques principalement au cours de déambulations collectives sur différents sites urbains comme les quartiers de la Défense, de Montparnasse, du Trocadéro et du Champs-de-Mars, ou bien dans les souterrains des $12^{\mathrm{e}}, 13^{\mathrm{e}}, 14^{\mathrm{e}}, 15^{\mathrm{e}}, 16^{\mathrm{e}}$ et $20^{\mathrm{e}}$ arrondissements.

\section{TABLEAU 2}

\section{Les lieux de pratique, ressemblances et dissemblances}

\begin{tabular}{|c|c|c|c|}
\hline & Description des lieux & $\begin{array}{l}\text { Nature } \\
\text { du lieu }\end{array}$ & $\begin{array}{l}\text { Modalités } \\
\text { de déplacement }\end{array}$ \\
\hline $\begin{array}{l}\text { Spéléologie } \\
\text { urbaine }\end{array}$ & $\begin{array}{l}\text { Grand Réseau du XIVe } \\
\left(\mathrm{XIV}^{\mathrm{e}}, \mathrm{XV}^{\mathrm{e}} \text { nord, } \mathrm{V}^{\mathrm{e}}, \mathrm{VI}^{\mathrm{e}}\right): 100 \mathrm{~km} \text {. } \\
\mathrm{XIII}^{\mathrm{e}} \text { arrondissement }: 25 \mathrm{~km} \text {. } \\
\mathrm{XVI}^{\mathrm{e}} \text { arrondissement (carrières } \\
\text { de Chaillot, réseau de Passy): } 7 \mathrm{~km} \text {. } \\
\text { XII }{ }^{\mathrm{e}} \text { arrondissement: } 200 \mathrm{~m} .\end{array}$ & Caché & $\begin{array}{l}\text { Mobile } \\
\text { Secret } \\
\text { Déambulations } \\
\text { souterraines }\end{array}$ \\
\hline
\end{tabular}

\begin{tabular}{|c|c|c|c|}
\hline Base-jump & $\begin{array}{l}\text { Tour Montparnasse }(210 \mathrm{~m}) \\
\text { Tour Eiffel }(300 \mathrm{~m})^{*} \\
\text { Arche de la Defense }(112 \mathrm{~m})\end{array}$ & Caché & Immobile \\
\hline Parkour & $\begin{array}{l}\text { Lisses (Evry) } \\
\text { Centre de Paris: } 13^{e} \text { arrondissement } \\
\text { à côté du quartier de Bercy }\end{array}$ & Visible & $\begin{array}{l}\text { Mobile } \\
\text { Fluide } \\
\text { Déambulations } \\
\text { dans la rue }\end{array}$ \\
\hline Golf urbain & $\begin{array}{l}\text { Champ de Mars ( } 7 \text { e arrondissement) } \\
\text { Parc de la Villette } \\
\text { Parc de la Defense }\end{array}$ & Visible & $\begin{array}{l}\text { Mobile } \\
\text { Fluide } \\
\text { Déambulations } \\
\text { dans la rue }\end{array}$ \\
\hline
\end{tabular}

* Les pratiquants sautent habituellement entre le second étage (115 mètres) et le troisième étage (275 mètres), ce qui représente une chute de trois ou quatre secondes avant l'ouverture du parachute. 
Voyons maintenant comment ces lieux sont explorés par les communautés pratiquantes mais surtout, comment ces acteurs urbains - en se représentant différemment l'espace parisien - font le choix, d'une part, de se concerter avec les institutions de tutelle et, d'autre part, de refuser toute concertation. La pratique sur des espaces cachés est alors de rigueur.

\section{Les «explor-acteurs» de la cité Se représenter la ville de Paris}

Les discours autour de «la» ville sont prépondérants dans la totalité des entretiens réalisés à la fois in situ et distanciés. Prolongeant le travail de De Certeau ici, se pencher sur les pratiques de l'espace permet de laisser parler les «pratiques microbiennes, singulières et plurielles» (1990, p. 145) plutôt que de traiter de l' «unité relevant d'une rationalité urbanistique» (p. 142). Les pratiques urbaines sont intiment liées à la nature de l'espace sur lequel une action motrice est engagée. De cette relation étroite entre le corps et l'espace se dégage une représentation particulière. Contrairement à ce que l'on pourrait penser, les pratiquants urbains entretiennent une relation intime avec leur habitat, avec leur ville de résidence:

J'ai toujours vécu en ville. C'est mon univers naturel, c'est là que je me sens bien, et en particulier dans ma ville. Quand je pars en vacances, c'est avant tout dans des villes ou pour visiter des restes de villes. Pour moi, la ville c'est Paris. Pas la banlieue. La banlieue a un statut à part. Peut-être parce que j'y ai vécu mon adolescence en rêvant de m'en échapper pour aller vivre à Paris. Les villes de province sont des villes mais pas «la ville». Peu de villes méritent cette appellation. Manhattan, Istanbul, Moscou, Le Caire, par exemple. Il faut un caractère universel, un caractère grouillant, un caractère éternel. (Spéléologue urbain, Paris.)

«À la fois machinerie et le héros de la modernité», la ville s'organise sur deux versants: le lieu du «pouvoir panoptique» (De Certeau, 1990, p. 145) urbanisation, stratégies socio-économiques - et le lieu des «transformations et appropriations [et de] mouvements contradictoires » (p. 144). Comment ces deux organisations cohabitent-elles au quotidien? Ce premier axe a émergé au fur et à mesure des rencontres effectuées sur ces terrains particuliers. Il fait écho aux relations complexes qui existent entre la qualité du cadre de vie, les politiques urbaines, culturelles ou sportives, les pratiques sociales et les cultures urbaines. La relation homme - ville, dans sa dimension sociale, sensible, culturelle ou même sanitaire, demeure centrale à cette première dimension. Pour illustrer nos propos, cet enquêté livre sa version concernant la double organisation urbaine: elle est - «un lieu sans vie» - et ce qu'elle permet de faire - «un bon délire entre potes»:

J'aime déjà le cadre... la ville... la nuit, c'est tranquille. C'est pas comme en journée il n'y a pas de bruit de fond ni de foule... Il y a aussi les rencontres avec les passants, qui sont le plus souvent cool... Mais c'est 
surtout un bon délire entre potes, l'occasion de passer une soirée autrement que enfermés dans un bar... Sans oublier que c'est une autre forme de grimpe avec des mouvements athlétiques et souvent déroutants, ce type de grimpe se rapproche plus du site naturel que de la salle et en soi... c'est un plus pour tous ceux qui habitent loin des falaises! Et puis la ville... oui, ben, c'est ça en fait [rires]. Les villes sont devenues des pièges qui assignent les corps à des postures rigides, qui canalisent les déplacements pour accélérer les flux de la périphérie-dortoir au centrebureau [rires]. Les villes, ce sont de multiples territoires et de multiples frontières visibles ou invisibles. La ville n'est pas habitée, elle est subie par ceux qui y habitent: des lieux sans vie, des vies sans lieux. (Explorateur, randonneur et grimpeur urbain, Paris.)

La ville permet encore de pratiquer chez soi, «at home», en dehors des équipements sportifs aménagés en zones urbaines et périurbaines (Augustin, 2001). Cette pratique de la ville en dehors des temps d'usage, la nuit notamment, devient un succédané du grand air des pratiques outsiders de pleine nature. Les pratiquants le vivent pleinement comme une possibilité de plus, un échappatoire au rythme effréné du jour, doublé d'une appropriation créatrice. Prenons l'exemple du street golf. La présence de parcours de golf en zone périurbaine et l'éloignement géographique - avec les contraintes que cela suppose - restreignent la localisation de la pratique. La pratique du street golf illustre une délocalisation de ces équipements traditionnels: «c'est simple et pratique, pas besoin de se taper des heures de caisse pour taper des balles! Et puis tu n'es pas limité à 18 trous, puisque le parcours urbain est infini!» (street golfeur). Dans ce cas de figure, la «dialectique ici-ailleurs » (Bourget, Moreux et Piolle, 1994) incarne pleinement la recrudescence des activités récréatives urbaines où l' «ailleurs» réside dans une construction sociale de sens, élaborée de manière compensatoire en réaction à la ville «rigide». Dès lors, la configuration spatiale du terrain de jeu urbain procure des sensations différentes pour ce pratiquant:

Le plaisir que tu éprouves quand tu es sur un parcours n'est pas le même que celui que tu éprouves dans la rue. Le terrain est différent, le parcours aussi... Quand je suis dehors, c'est l'éclate, la déchéance! Dans la rue, on a la sensation d'être borderline. Tu dois prendre en compte les gens, faire attention aux voitures... Ca te permet de casser les barrières du golf classique.

La personnalisation de la ville vient donc s'opposer aux espaces conçus, aux temps balisés. Des producteurs aux planificateurs de la ville, tous participent à la production d'une culture. La ville - à l'échelle globale - peut alors devenir modelable en fonction de sa propre représentation de l'espace. C'est ainsi que le grimpeur interviewé définit son rapport à la ville: une relation «piégée», nous dit-il, par un ensemble d'usages fonctionnels: y habiter, y circuler, y travailler. À travers ce discours, le pratiquant illustre la représentation apollinienne des grandes villes. En d'autres termes, l' «habitant du monde apollinien doit être transparent, distant» (Moreau et Sauvage, 2006, 
p. 70). De la sorte, il y a peu d'espaces propices aux liens interpersonnels et aux rencontres entre citadins, précise le pratiquant, elle "n'est pas habitée» mais plutôt occupée de manière rationnelle. La sociabilité est-elle coupée pour autant? La question de l'expérience spatiale est alors primordiale ici. Ainsi, des usages différenciés viennent contrecarrer cette vision «rigide» de la ville.

Car, la ville peut aussi être «molle» et «souple ${ }^{10} »$, c'est-à-dire modelable et négociable en fonction de la diversité des pratiques individuelles et collectives: «la ville est souple, elle attend de l'empreinte d'une identité [...] elle vous invite à la renouveler, à la consolider dans une forme que vous pouvez vivre, vous aussi. Décidez de ce qu'elle est, et votre propre identité sera révélée ${ }^{11} »$. De là surgissent des espaces de sociabilités ${ }^{12}$, de pratiques, et de représentation, bref, des lieux qui font lien!

C'est la ville qui nous motive avant toute chose... on y habite donc bon... on a un regard sur elle que d'autres n'ont pas forcément! Quand l'urbanisme sert un agencement savant de la ville à des strictes fins d'utilité sociale, des résistances se déploient, certaines concernent de nouveaux modes de penser l'architecture et l'urbanisme, d'autres, la manière même d'y habiter et de s'y déplacer! Et puis... j'aime les défis, les rencontres inattendues... et puis il y a plusieurs points [rires] D'abord... l'homme a envahi la nature par le béton... il n'y a plus rien à explorer qui a déjà été fait. Nous, on découvre notre monde sous un regard différent. Ensuite, le grimpeur est un être solitaire, s'enfermant dans des salles ${ }^{13}$, s'isolant au milieu des parois... La grimpe urbaine permet d'amener et de faire découvrir ce noble sport aux néophytes! L'ambiance de la nuit aussi... c'est tellement différent des rues commerçantes bondées de l'après-midi, les gens sont paisibles et nous aussi... Les rencontres sont inattendues et réservent toujours des surprises! (Explorateur, randonneur et grimpeur urbain, Paris.)

Par opposition aux non-lieux urbains - les espaces fonctionnels par exemple-, ces espaces de pratiques deviennent des «lieux anthropologiques» (Augé, 1992). Situés au centre d'une dynamique identitaire qui mêle l'individuel et le collectif, le lieu pratiqué physiquement, directement, par corps donc, acquiert du sens dans cet espace approprié et transformé. Les lieux sont « ouverts» et propices aux rencontres hasardeuses: rues, parcs, places, etc. A contrario, dans le cas où le lieu est principalement fermé (spéléologie urbaine), la dynamique identitaire est également centrale car les frontières du lieu sont nettement plus distinctes. Les groupes de base-jumpers attribuent à certains «spots » connus des caractéristiques identitaires, relationnelles et historiques. Ils constituent en quelque sorte le langage, les signes et, finalement, le sens de ces «lieux anthropologiques». De cette manière, la ville permet une autre issue: le détournement des espaces. Pour les enquêtés, les architectures urbaines incarnent une série d'obstacles qu'il est bon d'apprendre à franchir, ou dont il convient de s'affranchir. Cet apprentissage mobilise 
à la fois des qualités mentales et physiques. Par exemple, comme espace de corporéité, le parkour illustre très bien la catégorie analytique de l'«outilcorps » et du «corps-signe» (Berthelot, 1983). En tant qu'analyseur du social et du caractère sensible qui fait du corps une marque à la fois individuelle et collective, le parkour marque alors la réappropriation des corps urbains et les usages que les «traceurs» en font pour chevaucher les architectures urbaines. Le corps en mouvement figure alors un instrument avec lequel ils aménagent leur milieu de vie.

Une bonne façon d'entraîner son esprit à refuser les limites imposées par la société est simplement de chercher de nouvelles façons d'utiliser les obstacles et de les essayer. Si vous pouvez utiliser un obstacle d'une façon complètement opposée à celle pour laquelle la société l'a conçu, c'est un signe pour votre esprit qu'il y a des choses que la société oublie et cela vous force à considérer les erreurs dans les coutumes sociales [...] Quand votre esprit sera libre de l'influence de la société, vous noterez qu'il y a beaucoup de choses qui reposent sur l'acceptation des règles sociales par les gens [...] Lorsqu'on pratique le parkour, il est aisé d'oublier combien de limitations les autres placent sur le mouvement. Penser par soi-même plutôt que d'accepter ce que quelqu'un d'autre vous a dit nécessite plus d'effort mental. Le parkour a pour but d'entraîner votre esprit à fonctionner à travers l'action du mouvement à chaque fois que vous aurez besoin de vous déplacer au lieu de tomber perpétuellement dans la même routine. Chaque fois que vous vous déplacez, vous devez évaluer ce qui vous entoure pour déterminer la meilleure route pour aller à un endroit. (Récit de pratique, parkour.)

Pour résumer, la ville est imaginée de telle sorte qu'elle est personnalisée à travers la pratique spatialisée de ses architectures. Peut-on alors logiquement postuler que la ville, comme milieu de vie, agit sur les comportements individuels, mais qu'en retour, les pratiques de ces acteurs urbains permettent une construction individuelle et une auto-organisation de son milieu de vie? L'usage de la ville est ainsi questionné. Peut-on jouer de manière libre dans une ville? Le symptôme de l'adaptation de l'individu à son milieu naturel - le béton - convoque la notion de biotope. Dans le cas présent, le biotope serait alors le béton et suppose par là même, que les pratiquants en s'y adaptant le transforme de deux manières: de manière cachée ou bien visible.

\section{Les pratiques cachées et visibles de l'espace parisien}

À partir des représentations des acteurs et des pratiquants sur leur ville d'habitat, il est intéressant de voir comment la représentation va guider la pratique des espaces usés, traversés ou abandonnés dans Paris. D’une manière générale, la ville relève ainsi d'une valeur d'usage - ce qui accorde la priorité au social et à la socialité - et non plus uniquement d'une valeur d'échange (Simmel, 2004; Lefebvre, 2000). Les espaces de représenta- 
tions correspondent ici aux espaces de la ville transformés et appropriés par les communautés pratiquantes. Nous en distinguerons deux sortes. Premièrement, nous avons identifié les espaces de pratique qui ne se voient pas, car ils sont essentiellement cachés des citadins, à l'image des niches urbaines. Les toits d'immeuble et les souterrains servent alors à la pratique du base-jump urbain et de la spéléologie urbaine. Deuxièmement, nous avons dégagé une catégorie d'analyse centrée cette fois autour des espaces de pratique dits «visibles». Ils sont situés au cœur de l'activité urbaine, des citadins et sont donc visibles de l'extérieur. Le parkour et le street golf jouent notamment là-dessus pour renforcer leurs communications auprès des médias culturels et des organismes de régulations urbaines (à travers les politiques urbaines et sportives).

\section{Des espaces qui ne se voient pas}

Dans un premier temps se dégage une pratique ludosportive des espaces urbains où des «praticiens jouent des espaces qui ne se voient pas» (De Certeau, 1990, p. 141). C'est le cas plus particulièrement de la spéléologie urbaine et du base-jump urbain. Ces deux activités n'ont pas les mêmes modalités de pratique, toutefois, nous les rapprochons l'une de l'autre car elles se situent dans les interstices de la légalité et jouent très souvent avec les aspects juridiques qui permettent de différencier les espaces publics des espaces privés (le toit de l'immeuble, les souterrains des habitations, les zones souterraines fermées et protégées de toute intrusion humaine). Pour mieux saisir cette particularité, voyons comment une spéléologue urbaine présente son terrain de jeu, les souterrains:

il y avait un peu... une petite part d'inconnu... un petit peu magique dans le fait de progresser dans les galeries comme ça...dans... un grand labyrinthe...puis y a un petit côté confiné... un petit côté... petit côté... hmm... enfin,... c'est mon avis à moi... mais un petit côté protecteur... on se sent bien...dans ces galeries... avancer de salle en salle....(Femme spéléologue.)

Cette évocation féminine de la protection est-elle particulière? Elle est peut-être caractéristique d'une autre manière d'aborder cette activité largement masculine. En fait, les spéléologues urbains valorisent plutôt le glissement de tout le corps vers le «monde du dessous» (expression indigène); ils visent à se démarquer du «monde du dessus», royaume de l'organisation observatrice et des structures du pouvoir (Foucault, 1975).

Pour nous, tout cela représente surtout un moyen de s'exprimer sur un territoire de non-droits, où la loi ne s'applique pas. Et là ça change toute la donne... Crois-moi [...] Je t'ai dit que c'est un territoire de nondroits, maintenant rien ne dit que nous faisons nos propres lois... c'est justement ce que nous ne voulons pas... c'est ce que l'on cherche dans ces dessous.... Mais bon, la présence symbolique de la brigade policière nous rappelle que nous sommes là uniquement car rien n'est fait pour 
nous foutre dehors... ils nous tolèrent comme ils disent. L'amende s'élève à 25 euros maintenant pour les amendes piétonnes. C'est leur façon de nous montrer que nous ne sommes pas les seuls maîtres à bord... mais ça c'est une autre histoire... [rires]. (Spéléologue urbain.)

Pour autant, le retour dans le «monde du dessus» n'est pas non plus déprécié comme on peut se l'imaginer ex ante. Les données recueillies sont unanimes. La valorisation des souterrains et de leurs caractéristiques austères est vécue comme un complément spatial, un opérateur qui permet à ces ruses urbaines d'être l'élément qui valorise un aller-retour entre les espaces $\mathrm{du}$ dessus et ceux du dessous. Ce double trajet descente/remontée permet d'accorder une signification aux souterrains pratiqués. Pour preuve, les spéléologues urbains savent reconnaître les bienfaits de la lumière et, ainsi, redevenir visibles devant les foules urbaines. Pour justifier de cet état de conscience, certaines sensations sont alors valorisées qui soulignent l'importance du seuil à franchir entre l'espace de leur pratique et l'espace public:

Quand tu ressors de là, t'es content de voir la lumière... et t'es content d'entendre tous les petits bruits de l'extérieur en fait [rire].

Quand on sort du tunnel et qu'on découvre... le... [pause]... qu'on redécouvre le... ben... la nature... la lumière... le chant des oiseaux [rire]...autre chose que le clapotis des bottes dans l'eau et.... des couloirs à perte de vue... ou de non-vue... Ce moment où on sort du tunnel... lui il est vraiment magique....

La «ruse» consiste aussi à pratiquer les espaces qui ne se voient pas, nous l'avons dit, mais de manière discrète, souple et légère. Les pratiquants espèrent défendre le caractère fondé de leur démarche et de leurs randonnées illégales. Ils les légitiment en quelque sorte:

Dans la plupart des cas, c'est à l'abandon total qu'on se faufile discrète-
ment $\quad$ [rires]... pour accéder à l'intérieur.... Cela ne signifie pas
qu'il faut opérer le plus souvent en toute illégalité ou légalité vis-à-vis
des institutions... mais bon... moins y a de traces de notre passage et plus
le site sera conservé en l'état.... Et puis aussi... on ne vole pas.... On ne
tague pas, on ne dégrade pas... Il ne faut surtout pas oublier que d'autres
visiteront ce site après toi et voudront aussi en profiter.... Tes explorations
doivent être faites sur la pointe des pieds [rires]... Les photos prises
pendant les explorations servent aussi à pérenniser l'image de chaque site
et non à attirer le plus possible de casseurs... Si tu respectes ces règles
alors tu fais partie de la communauté des explorateurs [rires]....

En quoi consiste la pratique d'une espace qui ne se voit pas? Elle est à la fois une recherche d'inédit, une "exploration», comme le déclare un spéléologue urbain; elle est aussi une mise en retrait des pratiques plus courantes, une manière de se "protéger» contre autrui ou, encore, de se représenter secrètement sur des territoires de «clandestinités urbaines» (Aprile 
et Retaillaud-Bajac, 2008): «Je ne me retrouve pas dans une structure sociale prédéterminée. Je revendique une certaine liberté qui ne me semble accessible que dans quelque chose de non régulée.» (Base-jumper urbain.)

Chez ces deux communautés pratiquantes (spéléologie urbaine et basejump), la pratique ludosportive met en forme une culture du secret (Lebreton et Héas, 2007) où l' «esprit de groupe » est très prégnant:

Ce qui m'a marquée c'est qu'en bas les gens sont [pause]... pas tous les mêmes a priori. Mais ils ont tous le même uniforme... on a un peu le même langage... on est tous à peu près pareil quoi... bon... mis à part des histoires de caractère ou des choses comme ça... mais on se ressemble tous des fois... on est très surpris de voir qu'on aime bien discuter, fréquenter quelqu'un en dessous... quand on l'aperçoit en surface, on s'aperçoit qu'on n'aurait pas eu l'idée de lui dire bonjour ou d'être ami avec. (Femme, spéléologue urbaine.)

Cet «esprit de groupe» traduit alors un entre-soi qui délimite des frontières distinctes. La pratique de l' «espace qui ne se voit pas» se comprend comme des niches sociales où la créativité et le ludisme gagnent sur l'enfermement dans un espace dominant:

Quand on descend en sous-sol... c'est quand même un peu aussi... pour fuir le monde... on n'est pas particulièrement ravi de voir des foules de gens qui débarquent... des groupes de 20,25 personnes.... (Spéléologue urbain.)

Un espace de liberté gagné sur l'enfermement urbain [car]l'immeuble devient un immense promontoire offrant aux uniques base-jumpers des parties inexploitées de cette ville constamment étriquée. Le fait de pouvoir sauter d'immeuble nous fait découvrir la troisième dimension de cet espace qui nous entoure, cet espace qu'avant notre premier saut [...] nous considérions que comme plat. (Base-jumper urbain.)

Leur cadre d'expérience physique alternative, la solidarité et la convivialité sont de mises. Dans un sens, cette pratique particulière de l'espace se rapproche des «hétérotopies» de Foucault, ces «lieux dessinés dans l'institution même de la société, et qui sont des sortes de contre-emplacements, sortes d'utopies effectivement réalisées dans lesquelles les emplacements réels, tous les autres emplacements réels que l'on peut trouver à l'intérieur de la culture sont à la fois représentés, contestés et inversés, des sortes de lieux qui sont hors de tous les lieux» (Foucault, 1984).

Sous cet angle, le «droit à la ville» est donc bien plus qu'une simple utopie puisqu'il s'inscrit matériellement sur un «lieu», transforme alors la vie urbaine caractérisée par une absence de «lieux» identitaires et sensitifs, en une urbanité ludique et sensationnelle:

On marche beaucoup... euh... on se pose... très souvent... et... euh... quand on se pose... ben... on pique-nique... c'est vraiment ça... le piquenique... on sort, ses bières... euh... ses biscuits.... C'est plutôt le règne de l'austérité... le... dans les catas c'est le dénuement complet. Y a rien... 
y a des pierres... y a de l'eau... et des murs... ce n'est pas une prison parce que... on est libre... dedans... c'est la liberté... mais c'est le dénuement total. (Randonneur, explorateur et grimpeur urbain.)

Nous vivons dans la ville et la subissons... coincés dans les chemins tracés par les architectes bâtisseurs. L'exploration urbaine consiste à franchir ces limites dessinées par d'autres... enjamber une barrière, franchir une porte, ramper dans un tunnel, ouvrir une trappe.... En fait, toutes ces approches et les recherches qui conduisent à la partie utilitaire de la ville... souvent très esthétique.... Des endroits où vous n'êtes pas supposé aller et où vous quittez la partie toute tracée pour explorer un nouveau monde, celui de l'abandon et du merveilleux.... Après... libre à vous de choisir le lieu le plus propice à vos excursions urbaines. Le choix est immense, cela va du tourisme industriel aux toitures zinguées en passant par le petit théâtre de quartier oublié ou les immeubles décrépits et pourrissant au milieu de la ville... à l'abri des regards, isolés du monde normal, je dirais.... C'est le dernier pas qui vous fait basculer d'un monde à l'autre.... (Spéléologue urbain.)

Enfin, logiquement, cette pratique de l' «espace qui ne se voit pas» nécessite de la part des acteurs une organisation de «contre-pouvoirs» où le secret, la discrétion et la négociation avec les forces de l'ordre sont très présents. Deux déclarations de pratiquants illustrent ce point. La première précise comment une communication souterraine prend effet entre les pratiquants. Elle a été mise en place par et pour les randonneurs souterrains: «Il existe des coutumes propres aux habitués qui se sont développées avec le temps: des boîtes aux lettres particulières ${ }^{14}$, des salles où l'on est sûr de rencontrer des gens si l'on descend seul. Où on peut passer la nuit à refaire le monde en partageant une boisson.» (Spéléologue urbain.)

Ces pratiques mobilisent des réseaux de sociabilité très actifs. La communauté regroupe des «êtres qui souhaitent établir exclusivement entre eux des actions réciproques entièrement pures» (Simmel, 1981, p. 128). La caractéristique majeure de ces cultures urbaines d'un «nouveau» genre est que le mode de socialisation est exclusivement communautaire.

Si on est prêt à être base-jumper, c'est la communauté qui s'ouvre à nous [...] On ne se déclare pas base-jumper du jour au lendemain, on vient nous chercher et on dit: toi, tu as les compétences pour le devenir, on va te former, on va t'encadrer. Ça ne se fait qu'à cette condition. (Base-jumper.)

Pour rendre compte du trajet effectué dans la ville, de haut en bas, les base-jumpers et les spéléologues urbains pourraient comparer leurs activités à la mythique «chute d'Icare» et s'interroger sur les «retombées»: «faudra-t-il ensuite retomber dans le sombre espace où circulent des foules qui, visibles d'en haut, en bas ne voient pas?» (De Certeau, 1990, p.140.) Êtres invisibles, momentanément, que ce soit sur le haut ou le bas, dans le «monde $d u$ dessus» ou le «monde du dessous», les pratiquants interrogés peuvent 
être décrits comme des «êtres [qui] errent dans la nature surnaturelle, dans ce no man's land du temps [...] en attendant le retour vers le monde de la société et ses obligations» (Jeu, 1977, p. 37.)

\section{Des espaces qui se donnent à voir!}

Nous venons d'exposer les modalités de la pratique de l'espace qui ne se voit pas. Elles illustrent alors le versant de la différenciation sociale où un groupe d'individus refusent de voir leur pratique s'élargir au-delà de leur propre sphère communautaire. À l'inverse, la seconde logique d'actions repérée nous montre des groupes d'individus qui souhaitent élargir la sphère de pratiquants et acquérir une reconnaissance du grand public ${ }^{15}$.

La notion de «pratique de l'espace» est encore présente, mais sur la base de visibilité. À la question initiale, que signifie pratiquer la ville, les justifications avancées par les pratiquants renvoient «à une forme spécifique d'opérations (des «manières de faire»), à «une autre spatialité» (De Certeau, 1990, p. 142) ponctuée par une expérience anthropologique significative du «droit à la ville». Nous avons pourtant distingué deux opérations, l'une qui échappe à la visibilité, nous venons de le voir, et une autre qui se confond avec les pratiques ordinaires de la ville: marcher, courir, flâner, etc. Là encore, le «droit à la ville» est commun à ces pratiquants mais exercé de manière différente:

Les signalisations et les panneaux nous dictent inconsciemment ou consciemment notre chemin. L'urbanisme sert un agencement savant de la ville à des strictes fins d'utilité sociale. De ce fait, des résistances se déploient, certaines concernent de nouveaux modes de penser l'architecture et l'urbanisme, d'autres la manière même d'y habiter et de s'y déplacer. Qui n'a pas eu envie à un moment ou l'autre de braver ces interdits et d'aller là où bon lui semble. Peu de gens prennent l'initiative de redécouvrir ou d'utiliser la ville autrement. (Récit de pratique,parkour.)

Ce droit est mis en application de manière visible, là où d'autres auraient eu tendance à oublier l'effet de l'action des sociétés sur ce genre de données:

Le parkour nous fait utiliser l'instinct, les sens, des notions primaires du corps, des aptitudes que le corps n'a plus besoin d'utiliser dans notre société actuelle et encore moins dans les grandes villes comme ici... C'est pour ça que l'on cherche à savoir, à exprimer ces choses que l'homme a oubliées, voire effacées. (Parkour)

En revanche, la stratégie adoptée pour s'approprier la ville et ses espaces est quelque peu différente. En effet, la visibilité des espaces pratiqués montre que la «clandestinité» n'est pas de mise ici. Bien au contraire, dans la pratique du street golf et du parkour, des opérations de mixité culturelle pointent. Dès lors, la pratique s'affiche aux yeux de tous car l'espace de jeu 
est visible: «C'est simple et pratique. Pas besoin de se taper des heures de caisse pour taper des balles! Et puis tu n'es pas limité à 18 trous, puisque le parcours est infini» (street golfeur).

Les pratiques se déroulent alors sur des mobiliers et des architectures urbaines qui se donnent à voir. Plutôt que de parler d'une requalification de ces mêmes espaces, nous lui préférons la première expression. En effet, cela sousentend que le mobilier pratiqué et fréquenté est rendu visible par le jeu qui s'y déroule, d'une part, puis contribue à exposer les corps en action, d'autre part.

Pour moi, le parkour, c'est un art qui permet de mieux se connaître, d'échapper à cette prison, les murs. Pouvoir être libre. Il n'y a pas forcément de règles précises, mais la règle, je pense que c'est nos limites, ne pas faire n'importe quoi. Les obstacles qu'on peut rencontrer quand on trace sont d'une manière ou d'une autre nos ennemis... il faut les battre, les défier et surtout, réussir, du moins pour les obstacles qu'on peut rencontrer dans les rues.... Pour moi, les obstacles «vivants »... les arbres... les pierres... ne sont pas nos ennemis, mais plus des alliés, des personnes qu'on respecte... des chefs en quelque sorte, des chefs qui nous aident à avancer, à nous surpasser.... Le parkour, pour moi, n'est pas forcement un effort physique. On peut très bien pratiqués le parkour sans courir, ni sauter.... Le parkour est plutôt une manière de faire face à toutes les situations, de la vie de tous les jours. Tout le monde pratique le parkour sans même le savoir, pas forcement physiquement, mais tout le monde le pratique. (Parkour)

La pratique n'est pas illégale cette fois-ci. Elle n'a pas besoin d'être cachée ni dissimulée, bien au contraire, elle est communiquée:

E: Comment vous qualifieriez votre pratique? Une tendance? une activité alternative... underground? (Enquêteur)

e1: Oui et non.... Oui, car c'est une pratique tout juste tolérée et car on grimpe la nuit sans trop vouloir se faire remarquer... et non, car on communique beaucoup par l'intermédiaire de sites internet... d'associations.... (Grimpeur urbain, parkour)

e2: Je dirais oui, moi... mais qui sort de l'ombre pour se démarginaliser! En effet, on ne se cache plus, on va à la rencontre des passants.... On discute et on s'affiche à travers tous les supports modernes.... (Grimpeur urbain.)

Conscients de l'évolution grandissante des cultures de rues, ces pratiquants reconnaissent avoir le désir de se faire voir encore davantage:

Quand j'ai commencé l'urbain, je faisais ça pour le délire.... Grâce aux sites Internet j'ai pris conscience que ça branchait pas mal de monde. En plus, la pratique a déjà énormément évolué maintenant toutes les sorties sont organisées à l'avance... il y a des repérages... les gens ont un bon niveau aussi et les blocs sont de plus en plus intéressants.... Dans l'avenir, on va continuer dans cette voie-là, en essayant de prendre le moins de risques possible et en s'éclatant le plus possible. Je compte aussi beaucoup sur les associations pour sensibiliser le grand public.... (Grimpeur urbain.) 
À la différence des espaces sportifs, les espaces pratiqués ne sont pas destinés à un usage ludique et sportif. Les pratiquants détournent les architectures à leur profit mais pas seulement. Ce «droit à la ville»doit être accessible à toute la population urbaine. C'est la raison pour laquelle les espaces qui se donnent à voir ne sont pas seulement pratiqués pour la communauté pratiquante. Il y a dans cette démarche un projet commun - proche de la démarche citoyenne - qui vise à rendre visible ce qu'il est possible de faire:

Moi... j'en avais marre du boucan des salles pleines de poussière, marre de travailler jusqu'à point d'heure, ne pouvant plus aller grimper le soir... ou de me taper des heures de route le week-end pour aller toucher du caillou.... Quel plaisir de mettre ses doigts la toute première fois sur un mur qui n'est pas prévu à cet effet. Prendre du plaisir à lire l'architecture, sous un œil de sportif.... Les gens regardent mais ne savent rien de la richesse des ruelles. L'appel de l'inédit, ouvrir des premières voies m'est venu naturellement après avoir fait la découverte des berges de la Seine où des ponts et murs subsistent tant bien que mal.... J'ai donc eu envie d'aller voir toujours plus loin.... (Grimpeur et randonneur urbain.)

Pour autant, une «sportification» progressive concerne les pratiques d'espaces visibles (Lebreton, 2009). Elles sont donc vouées à être sans cesse redéfinies par les institutions sportives, économiques, politiques et policières, qui, en les redécouvrant, réorganisent les modalités et les frontières.

De la sorte, la ville «apparaît de nouveau comme lieu pratiqué » (De Certeau, 1990, p. 190) et est investie d'un esprit dionysiaque où la marche, la déambulation, la course, les sauts, la découverte de lieux, la légèreté, la dérision et l'ivresse des corps transportés dans la ville transforment le parcours ludosportif (la déambulation ludosportive) en un jeu codé et normé plutôt qu'une situation alternative ou marginale à proprement parler.

Pour ma part, je cherchais à me réapproprier mon environnement... je t'ai dit, je suis de la ville moi... donc si je veux m'amuser, il faut partir à la découverte de mon environnement quotidien. Avec le street golf, je ne le subis plus, je le détourne. Du coup,j’ai juste adapté mon sport principal à la rue! Les principes sont les mêmes que pour le golf classique sauf que les trous sont remplacés par des cibles urbaines.... (Street golfeur)

Finis les parcours imposés, on choisit ses cibles, en tenant compte de l'urbanisme, des règles de sécurité, de l'imprévu qui peut surgir, tout en gardant le goût du défi. Bref, s'adapter à l'environnement extérieur demande plus de concentration. Si on veut atteindre ses objectifs, il faut développer la patience, la maîtrise de soi et garder tous ses sens en alerte. Le street golf n'est pas seulement une activité physique, c'est aussi un véritable training mental. Et puis, là où trouver un partenaire pour s'entraîner sur le green peut relever du casse-tête, la version urbaine réintroduit un souffle de convivialité. Le street golf fonctionne essentiellement en communauté. (Street golfeur.) 
À l'image d'une «inscription du corps dans le texte de l'ordre» (De Certeau, 1990, p. 191) urbain, les conduites corporelles renouent avec un aménagement de leur milieu de vie par l'appropriation collective de lieux anthropologiques. Les bâtiments sont alors transformés objectivement sous l'effet de techniques corporelles: «Il y a pas beaucoup de falaises ici... et j'avais envie de faire autre chose que de grimper en salle et j'avais remarqué des structures assez intéressantes à côté dans des lieux en centre-ville ou en périphérie.... Un week-end, on a donc franchi le cap et essayé de grimper ces bâtiments.... (Grimpeur urbain et traceur.)

\section{Conclusion: vers une régulation urbaine des cultures sportives?}

En raison du développement important de ces pratiques physiques alternatives, la question de leur régulation demeure un point clé de certaines politiques urbaines et sportives. Cela renvoie à une forme de concertation entre les acteurs et les institutions. Cette régulation permet aux institutions politiques, aux organisations privées et aux acteurs sportifs de dialoguer pour trouver des réponses adaptées aux attentes de chacun: reconnaissance pour les uns et contrôle des pratiquants pour les autres. Ce mécanisme produit alors deux effets: la reconnaissance de certaines pratiques et l'exclusion d'autres. Le ludisme et la récréativité qui caractérisent ces loisirs urbains montrent aujourd'hui un double jeu entrepris par les acteurs de la ville (politiques, économiques et sociaux). D'un côté, on observe une régulation des espaces publics pour intégrer certains groupes sociaux à la cité et, de l'autre, l'exclusion et la marginalisation d'autres groupes. Les APS que nous qualifions d'alternatives le montrent très clairement. Prenons l'exemple des cultures urbaines. Certaines pratiques sont passées d'un étiquetage «sauvage», comme le hip-hop ou le skateboard l'ont initialement été, à une intégration aux politiques culturelles de la ville ${ }^{16}$. Cette transformation contribue à une reconnaissance, lente mais progressive, de ces cultures. Les créations de la fédération nationale des cultures urbaines (FNCU) à la fin des années 1990, puis dernièrement ${ }^{17}$, la fédération française des sports urbains (FFSU), l'attestent en partie. Les cultures urbaines sont reconnues mouvantes et polymorphes; elles prennent leur essor en marge des réseaux culturels officiels, elles sont souvent l'affaire d'autodidactes et se distinguent aussi par des manières d'être, des modes de communications et des règles sociales qui en font une matière à études socio-anthropologiques à part entière.

Il ne faut pas se méprendre sur la logique commune aux quatre groupes enquêtés. Les ressemblances culturelles mettant a priori les pratiques «alternatives » dans un même chapeau sont à nuancer. En effet, des dissemblances culturelles peuvent y être aussi repérées. La question des cultures et des sous-cultures sportives (Rinehart et Sydnor, 2003) rappellent que les sports 
participent des cultures populaires et tendent à perdre leur impact comme alternatives à la construction communautaire et personnelle. La ritualité est fortement concurrencée par le business sportif et sa logique marketing. Il est donc nécessaire de nuancer fortement aujourd'hui la compréhension de la manière dont ces pratiques sont réappropriées par les institutions urbaines et sportives. Nous l'avons vu, il se dégage deux dimensions significatives: un refus du conformisme pour la spéléologie urbaine et le base-jump urbain, d'une part, alors qu'une progressive «sportification» (Guttmann, 2006) est en marche pour le street golf et le parkour, d'autre part. Une certaine prudence est donc nécessaire quand il s'agit d'établir la sociologi(qu)e de ces activités physiques contemporaines.

Finalement, que révèlent ces activités hédonistes et libertaires inscrites au cœur des sociétés urbaines? Sont-elles totalement «nouvelles» ou marquent-elles plus simplement l'évolution progressive des sports vers des formes plus récréatives et plus ludiques? Doit-on parler précisément de différenciation sociale pour illustrer les transformations à l'œuvre dans l'espace social des sports? Les approches socioculturelles des pratiques sportives ${ }^{18}$, largement inspirées par les travaux de P. Bourdieu ${ }^{19}$, indiquent par exemple que la recherche de modes d'organisation à faibles contraintes ${ }^{20}$, la recherche de nouveaux cadres d'exercice, la désaffection des équipements sportifs traditionnels et la recrudescence des usages corporels visant à transformer les espaces de vie par la ruse et la tactique, ce sont les usages corporels décrits ici par exemple, deviennent des techniques dites de transposition ${ }^{21}$. Les modalités «libre» et «autonomie» forment la base du modèle participatif et hédoniste des activités physiques et sportives contemporaines et d'autant plus lorsque c'est l'espace urbain, et notamment la rue, qui détermine la pratique. Cette prospective alimente nos conclusions sur le développement de l'auto-organisation sportive et la diversification des pratiques et de leurs modalités en France, qu'elles soient institutionnalisées ou non.

\section{NoTES}

1. Acronyme pour désigner le saut en parachute de points fixes tels: Buildings, Antenna, Spin et Earth (BASE-jump). On distingue deux logiques de pratique, une dite naturelle (environnement montagnard) et une seconde urbaine (immeuble, tour).

2. Mélange de gymnastique et de course à travers les mobiliers urbains.

3. Notre recherche a reçu le soutien financier de l'ANR-08-VULN-001-PRASGEVU qui focalise précisément sur les vulnérabilités liées au genre dans les sports.

4. Thèse de doctorat de sociologie soutenue le 14 avril 2009 à l'Université européenne de Bretagne-Rennes II. "Faire lieu» à travers l'urbain. Socioanthropologie des pratiques ludosportives et auto-organisées de la ville. 
5. Nom donné aux pratiquants du parkour.

6. Pour un compte rendu du travail ethnologique, nous vous renvoyons à la publication de Lebreton et Héas (2007).

7. À la suite de Wacquant (2002), nous soulignons la nécessaire distinction entre une observation participante et une participation observante et inscrivons notre travail dans le premier cas. En effet, la logique heuristique a toujours prévalu sur la tentation de la pratique ludosportive et le souci de s'intégrer dans les groupes pratiquants en cultivant le culte du secret qui leur est largement inhérent (Simmel, 1911).

8. À notre connaissance, l'unique enquête sociologique de ce genre est portée par Glowczewski B. (1983). Les cataphiles - Mission anthropologique dans les souterrains de Paris. Paris, Librairie des Méridiens.

9. Par exemple Adamkiewicz, E. (1998). Les usages sportifs autonomes de la ville, Analyse des pratiques, aménagement et management des espaces. Thèse de doctorat. Université de Lyon 1, Villeurbanne. Calogirou, C. \& Touché M. (1995b). Rêver sa ville: l'exemple des pratiquants du skateboard. In Le Journal des Anthropologues, n ${ }^{\circ}$ 61-62, pp. 67-77. Chantelat, P., Fodimbi, M., \& Camy, J. (1996). Sports de la cité. Anthropologie de la jeunesse sportive. Paris:L'Harmattan. Duret, P., Augustini, M. (1993). Sports de rue et insertion sociale. Paris, INSEP. Pedrazzini, Y. (2001). Rollers et skateurs: sociologie du hors-piste urbain, Paris, L'Harmattan, coll. «Changement».

10. Raban, J. (1974). The soft city. Londres: Harvil Press.

11. "The city goes soft; it awaits the imprint of an identity. For better or worse, it invites you to remake it, to consolidate it into a shape you can live in. You, too. Decide who you are, and the city will again assume a fixed form around you. Decide what it is, and your own identity will be revealed, like a position on a map fixed by triangulation» Ibid., p.11.

12. Par sociabilité, nous nous référons aux travaux de Simmel sur le lien interpersonnel qui marque la relation basée sur le contact de proximité.

13. L'interviewé fait référence ici aux citadins qui pratiquent de l'escalade en milieu fermé (gymnase, mur d'escalade)

14. Référence à la présence de nombreux tracts au sein des galeries souterraines illustrant des sujets divers et variés.

15. Un article traitant tout particulièrement de ce point est actuellement en expertise. Lebreton, Routier, Héas et Bodin, 2010, «Cultures urbaines et activités physiques et sportives. Le "sportification" du parkour et du street golf comme médiation culturelle».

16. Nous pensons notamment aux prises en compte des sports urbains dans le cadre de rapports ministériels «Cultures urbaines», Rapport au ministre de la Culture et de la Communication, mars 2007.

17. Les statuts de la FFSU ont été déposés en janvier 2010 auprès du ministère français de la Santé, de la Jeunesse et des Sports.

18. C. Pociello, 1999. Sport et sciences sociales. Histoire, sociologie et prospective, Paris: Vigot.

19. P. Bourdieu, 1979. La distinction. Paris: Éditions de Minuit.

20. Ce que nous appelons les activités auto-organisées.

21. Pociello, op. cit. 


\section{BibLiOgRAPHIE}

APRILE, S. et RetAillaud-BAJAC, E. (2008). Clandestinités urbaines. Les citadins et les territoires du secret (XVI $I^{e}-X X^{e}$ siècles). Rennes: Presses universitaires de Rennes.

AtKInsOn, M. (2009). Parkour, anarcho-environmentalism, and poiesis. Journal of Sport \& Social Issues, 33( 2), 169-194.

AtKinson, M. et Young, K. (2008). Sport, deviance and social control. Champaign: Human Kinetics.

Augé, M. (1992). Non-Lieux, introduction à une anthropologie de la surmodernité. Paris: Seuil.

Augustin, J.-P. (2001). Espaces publics et cultures sportives. Geocarrefour, 76(1), 27-30.

BAvinton, N. (2007). From obstacle to opportunity: Parkour, leisure, and the reinterpretation of constraints. Annals of Leisure Research, 10(3/4), 391-412.

BECKer, H. (1985). Outsiders. Études de sociologie de la déviance. Paris: Métailié.

Berthelot, J.-M. (1983). Corps et Société. Problèmes méthodologiques posés par une approche sociologique du corps, Cahiers internationaux de sociologie, LXXIV, 119-131.

Bourget, M., MoreuX, C. et Piolle, X. (1994). Pratique de la montagne et société urbaine. Revue de Géographie Alpine, 82(4).

BOUvier, P. (2000). La socio-anthropologie. Paris: Armand Colin.

Bromberger, C. (2004). Les pratiques et les spectacles sportifs au miroir de l'ethnologie. In Dispositions et pratiques sportives (Société de Sociologie du Sport de Langue Française éd.), Paris: L'Harmattan, p. 115-128.

CAlogirou, C. (1999). Le parcours ou la conquête acrobatique de l'espace urbain: Entretien avec David Belle, fondateur du groupe Yamakasi. In Ville, école, intégration, $\mathrm{n}^{\circ} 116,97-112$.

Chantelat, P., Fodimbi, M., et Camy, J. (1996). Sports de la cité. Anthropologie de la jeunesse sportive. Paris: L'Harmattan.

Chevalier, S., et Privat, J.-M. (2004). Elias et l'anthropologie. Paris: CNRS Éditions.

De Certeau, M. (1990). L'invention du quotidien. Arts de Faire (tome 1). Paris: Gallimard.

Daskalaki, M., Stara, A. et Miguel, I. (2008). The 'Parkour organisation': inhabitation of corporate spaces. Culture and Organisation 14(1), 49-64.

Donelly, P. (1985). Sport Subcultures. Exercise and Sport Science Review, n¹3, 539-578.

DonNelly, P (1993). Subcultures in sport: Resilience and transformation. In A. Ingham etJ. Loy (dir.). Sport and social development: Traditions, transitions and transformations, Champaign: Human Kinetics, p.119-145

Elias, N. (1993). Engagement et distanciation. Paris: Fayard (1 ${ }^{\text {re }}$ édition 1983).

FOUCAULT, M. (1975). Surveiller et punir. Paris: Gallimard.

Foucault, M. (1984). Des espaces autres (conférence au Cercle d'études architecturales, (14 mars 1967). Dits et écrits, Tome IV, $\mathrm{n}^{\circ}$ 360, p. $752-762$, Paris: Gallimard. 
GEYH, P. (2006). Urban free flow: A poetics of parkour. Media culture journal 9(3), 4.

Guttmann, A. (2006). From ritual to record. The nature of modern sports [1978]. Traduction de T. Terret. Paris: L'Harmattan.

GlowCZeski, B. et MATTEUdi, J.-F. (1983). La cité des cataphiles, mission anthropologique dans les souterrains de Paris. Paris: Éditions de Minuit.

Hannertz, U. (1983). Explorer la ville. Paris: Éditions de Minuit.

HÉAS, S. et SAÏDI TH., (2000). Rapport d'enquête auprès des 309 associations sportives rennaises: profils des pratiquants, emplois offerts et utilisations des équipements, UFRAPS./Office des sports de Rennes, juin.

JEU, B. (1977). Le sport, l'émotion, l'espace. Paris: Vigot, édition 1984.

JoSEPH, I. (1998). La ville sans qualité. Lyon: Édition de l'Aube.

LAPLANTINE, F. (1996). La description ethnographique, Paris : Nathan.

LEBRETON, F. (2009). «Faire lieu» à travers l'urbain. Socio-anthropologie de pratiques ludo-sportives et auto-organisées de la ville. Thèse de doctorat de sociologie non publiée. Université européenne de Bretagne, Rennes II.

LEBRETON, F. et HÉAS, S. (2007). La spéléologie urbaine. Une communauté secrète de cataphiles. In Ethnologie française, 37(2), p.345-352.

LeFebVRe, H. (2000). La production de l'espace. Paris: Anthropos.

Mould, O. (2009). Parkour, the city, the event. Environement and Planning: Society and Space, 27, 738-750

MUKAMURERA, J. L. (2006). Des avancées en analyse qualitative: pour une transparence et une systématisation des pratiques. In Recherches qualitatives 26(1), 110-138.

OLIVIER DE SARDAN, J.P. (2008). La rigueur du qualitatif. Les contraintes empiriques de l'interprétation socio-anthropologique, Louvain-la-Neuve, Academia Bruylant.

OrtuZAR, J. (2009). Parkour or l'art du déplacement: A kinetic urban utopia. The Drama Review, 53(3), 54-66.

Ostrowetsky, S. (1996). Sociologues en ville. Paris, L'Harmattan.

Piette, A. (1996). Ethnographie de l'action. L'observation des détails. Paris: Métailié.

RINEHART, R. et SYDNOR, S. (2003). To the extreme: alternative sports, inside and out. Albany: State University of New York Press.

SANSOT, P. (1986). Les formes sensibles de la vie sociale. Paris: Presses universitaires de France.

SAnsot, P. (2004). Poétique de la ville. Paris: coll. Petite Bibliothèque Payot.

SAVILlE, S. J. (2008). Playing with fear: Parkour and the mobility of emotion, Social and Cultural Geography 9(8), 891-914.

SIMMel, G. (1981). Sociologie et épistémologie. Paris: Presses universitaires de France.

SIMMEL, G. (1996). Secret et sociétés secrètes. Paris: Circé.

SimMel, G. (1999). Sociologies. Étude sur les formes de la socialisation. Paris: Presses universitaires de France, Sociologies

Simmel, G. (2004). Philosophie de la modernité. Paris: Payot (2e édition).

Thompson, D. (2008). Jump City: Parkour and the traces. South Atlantic Quarterly 107(2) 251-263.

WheAton, B. (2004). Understanding lifestyle sports: Consumption, identity and difference. London: Routledge. 
Florian LEBRETON, Stéphane HÉAS et Michael ATKINSON

Urbanité ludique et cultures sportives.

Analyse de quatre pratiques physiques de l'espace parisien

\section{RÉSUMÉ}

La reconquête de sites urbains et périurbains s'inscrit dans l'évolution des cultures sportives contemporaines. Elle est marquée, entre autres, par l'émergence de pratiques ludosportives alternatives (spéléologie urbaine, parkour, street golf, etc.) qui ne s'inscrivent pas sur les espaces aménagés. Ces pratiques physiques, véritables jeux novateurs, élargissent l'éventail des pratiques culturelles et mobilisent ce que nous appelons une urbanité ludique. La réappropriation des espaces urbains suppose une adaptation des techniques corporelles et sportives usuelles (course, gymnastique, etc.) aux lieux pratiqués: rues, parcs, jardins, places, souterrains, immeubles. Cette réappropriation contribue fortement à développer une légitimité ludique et à souder les participants au sein d'une même communauté pratiquante et auto-organisée. L'identification et le «droit à la ville» analysés ici permettent de distinguer deux logiques d'actions: des pratiques ludosportives urbaines s'inscrivant sur des lieux qui ne se «voient pas»-invisibles; une seconde concernant les lieux qui se «donnent à voir» - visibles.

Mots clés: cultures urbaines, cultures sportives, pratique ludosportive, urbanité ludique.

Florian LEBRETON, Stéphane HÉAS and Michael ATKINSON

Playful Urbanity and sports cultures: Analysis of four physical practices of the Parisian space

Taking back urban and peri-urban sites is part of the evolution of contemporary sports cultures. It is marked by the emergence of alternate sportstainment practices (urban speleology, parkour, street golf etc.) that do not take place in dedicated venues. Such physical practices, truly innovative games, widen the array of cultural practices and mobilize what we have come to know as playful urbanity. In order for the reappropriation of urban spaces to take place, the body and sports techniques (running, gymnastics etc.) need to adapt to new locations: streets, parks, gardens, places, tunnels and buildings. This reappropriation strongly contributes to the development of a playful legitimacy as it unites participants into an active and self-organised community.

The identification and the right to the city present us with two distinct logics of action: urban sportstainment practices take place in locations that can not be seen (invisible) and in locations that can (visible).

Key words: urban cultures, sports cultures, playful urbanity 